\title{
Perforación radicular asociada al uso de microtornillos en el anclaje ortodóncico: Reporte de un caso
}

\section{Root perforation associated with the use of micro-screws in orthodontic anchorage: A case report}

\author{
Holmberg Peters Fdo', Holmberg Peters F², Zaror Sánchez $C^{3}$
}

\section{RESUMEN}

Este reporte de caso describe el tratamiento quirúrgico de un primer molar maxilar como solución a la perforación iatrogénica de su raíz mesiovestibular tras la colocación de un microtornillo para el refuerzo del anclaje ortodoncico en un paciente adulto. El objetivo de este caso clínico es dar a conocer una de las posibles complicaciones asociadas a la colocación de microtornillos interradiculares asociados al refuerzo del anclaje en ortodoncia, su diagnóstico y tratamiento.

Rev. Clin. Periodoncia Implantol. Rehabil. Oral Vol. 6(3); 138-141, 2013.

Palabras clave: Microtornillo, perforación radicular, radectomia.

\section{ABSTRACT}

The aim of this publication is to present one of the possible complications associated with the placement of interradicular miniscrews for the reinforcement of anchorage in orthodontics. This case report describes the surgical treatment of a maxillary first molar as a solution for the iatrogenic perforation of its mesiobuccal root after the placement of a microscrew for the reinforcement of the orthodontic anchorage in an adult patient.

Rev. Clin. Periodoncia Implantol. Rehabil. Oral Vol. 6(3); 138-141, 2013.

Key words: Microscrew, root perforation, radectomy.

\section{INTRODUCCIÓN}

Los microtornillos han revolucionado los tratamientos de ortodoncia, ya que con ellos se puede realizar el anclaje ortodoncico sin comprometer los dientes posteriores, siendo removidos una vez que termina su función ${ }^{(1)}$.

Si se realiza un adecuado estudio del caso, se tiene un dominio en la técnica y se lleva a cabo de manera precisa, es muy difícil que se presenten complicaciones. No obstante se reportan como principales dificultades la fractura del tornillo, daño de las estructuras anotómicas, mucositis y periimplantitis ${ }^{(1)}$.

En relación al daño de estructuras anotómicas, el trauma del ligamento periodontal o de la raíz del diente se produce durante la inserción del microtornillo. Las potenciales complicaciones de esta injuria incluyen perdida de la vitalidad pulpar; osteoesclerosis y anquilosis dental $^{(2)}$.

El trauma radicular sin compromiso pulpar parece no influenciar el pronóstico del diente. Las raíces dañadas por los microtornillos de ortodoncia han mostrado completa reparación del tejido dental y periodonto entre las 12 y 18 semanas posterior al retiro de este ${ }^{(2)}$. Sin embargo, si el cemento está dañado mecánicamente y la dentina está expuesta, células multinucleadas pueden colonizar las superficies desnudas, llevándose a cabo el fenómeno de reabsorción radicular(2).

La imagenología juega un rol fundamental para el diagnostico de las perforaciones radiculares. Sin embargo, las radiografías convencionales utilizadas frecuentemente para este propósito representan objetos de $3 \mathrm{D}$ con imágenes de $2 \mathrm{D}$ con una significativa superposición de estructuras y magnificación impredecible lo cual dificulta el diagnostico imagenológico. Es así como la tomografía computarizada permite una visualización real en 3D de las estructuras óseas y dentoalveolares. La tomografía digital volumétrica presenta similares virtudes que la tomografía convencional y adiciona una menor radiación del paciente ${ }^{(3)}$.

El objetivo de este reporte de caso es dar a conocer un caso de reabsorción radicular asociada a la colocación de un microtornillo como elemento de ayuda en el anclaje ortodóncico, las complicaciones en el diagnostico y posterior manejo de la perforación radicular.

\section{DESCRIPCIÓN DEL CASO CLÍNICO}

Paciente de sexo femenino, 31 años de edad cuyo motivo de consulta se asocia a dolor localizado en relación al primer molar superior izquierdo.

Dentro de la anamnesis la paciente relata haber recibido tratamiento de ortodoncia con aparatos fijos hace 2 años atrás. Como parte de la mecánica de tratamiento se insertaron 2 microtornillos, cada uno en posición mesial a dientes 1.6 y 2.6 respectivamente con el objetivo de reforzar el anclaje ortodóncico. Semanas después relata que comenzó a percibir dolor agudo localizado en el diente 2.6, razón por la cual su odontólogo tratante retiro el microtornillo. Como el dolor se mantuvo se le realizó una endodoncia en el molar. Sin embargo, las

1. Magíster en Odontología. Especialista en Periodoncia. Especialista en Ortodoncia y Ortopedia DentoMaxiloFacial. Universidad de La Frontera. Temuco, Chile.

2. Cirujano Dentista. Universidad de Antofagasta. Antofagasta, Chile.

3. Magíster en Epidemiología Clínica. Especialista en Odontopediatria. Departamento de Odontopediatria y Ortodoncia, Facultad de Odontología. Universidad de La Frontera. Temuco, Chile.

Correspondencia autor: Fernando Holmberg Peters. fr.holmberg@yahoo.com. Trabajo recibido el 28/10/2012. Aprobado para su publicación el 15/01/2013. 
molestias persistieron por lo que la paciente relata que se le realizó una cirugía exploratoria en la zona sin resultados positivos.

Al examen clínico se observa una fistula vestibular y recesión gingival en relación al primer molar superior izquierdo (Figura 1).

Al examen radiográfico se observaba un molar endodonticamente tratado y ausencia de lesión apical o pararadicular (Figura 2), motivo por el cual se complementa el examen con una tomografía digital volumétrica de la zona que fue concluyente en el diagnostico de granuloma apical de la raíz mesiovestibular del diente 2.6 y ausencia de la tabla ósea vestibular. A nivel del tercio medio de la raíz mesiovestibular se observa un área hipodensa de límites definidos, que compromete el tabique óseo interdentario y la raíz mesiovestibular, imagen sugerente con exorizálisis (Figura 3 ).

En la Figura 4 se observa la perdida completa de la tabla ósea vestibular en la raíz mesial, sin embargo, no es evidente la perforación radicular generada por el microtornillo y que es claramente observada en la tomografía digital volumétrica, lo cual puede explicar el no hallazgo de la lesión cuando se realizó la cirugía exploratoria.

Dado el tiempo de evolución de la lesión (24 meses) junto con la extensión de esta y posterior a la evaluación por un endodoncista se procede a realizar la radectomia de la raíz mesiovestibular del diente 2.6 (Figura 5). En la Figura 6 se puede observar la imagen postoperatoria inmediata.

En la Figura 7 se puede observar el control clínico y radiográfico a los 6 meses de realizado el procedimiento quirúrgico.

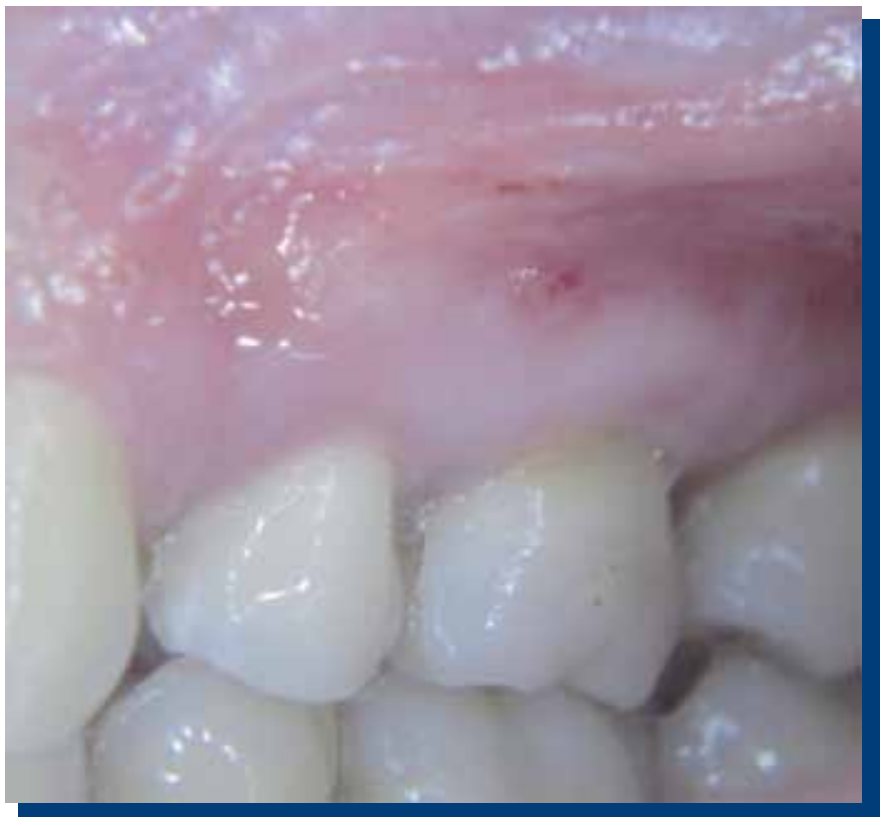

Figura 1. Examen clínico inicial.

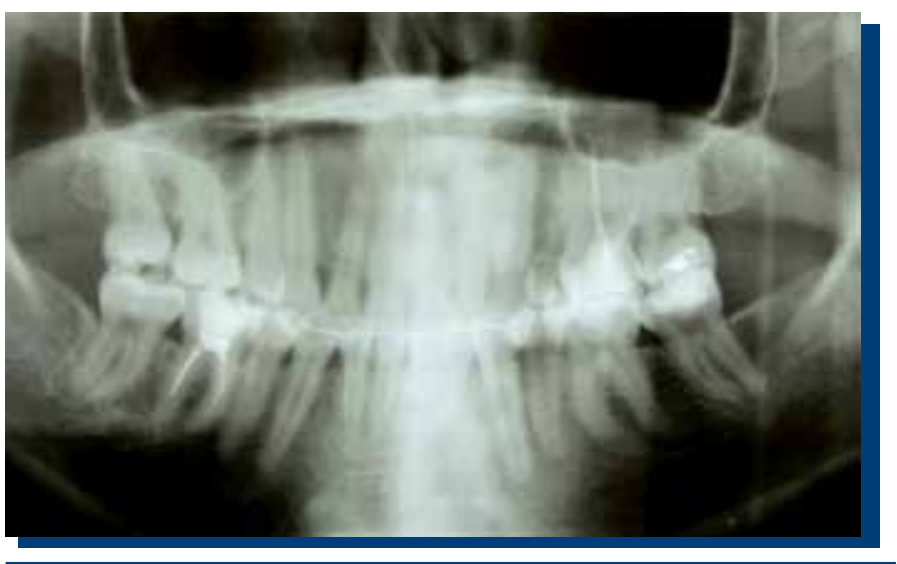

Figura 2. Radiografía panorámica inicial.

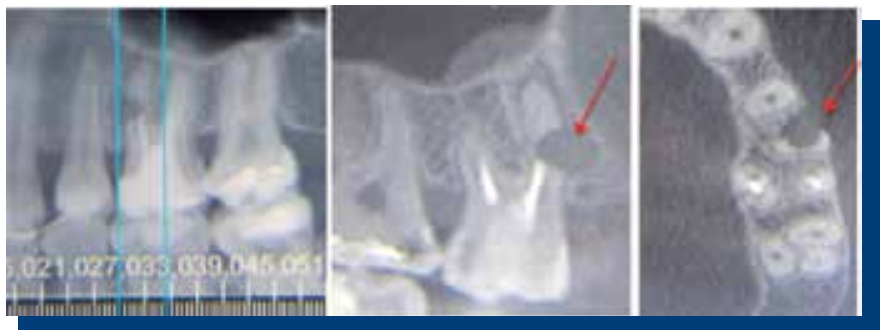

Figura 3. Tomografía digital volumétrica. Exorizálisis tercio medio raíz mesiovestibular diente 2.6.

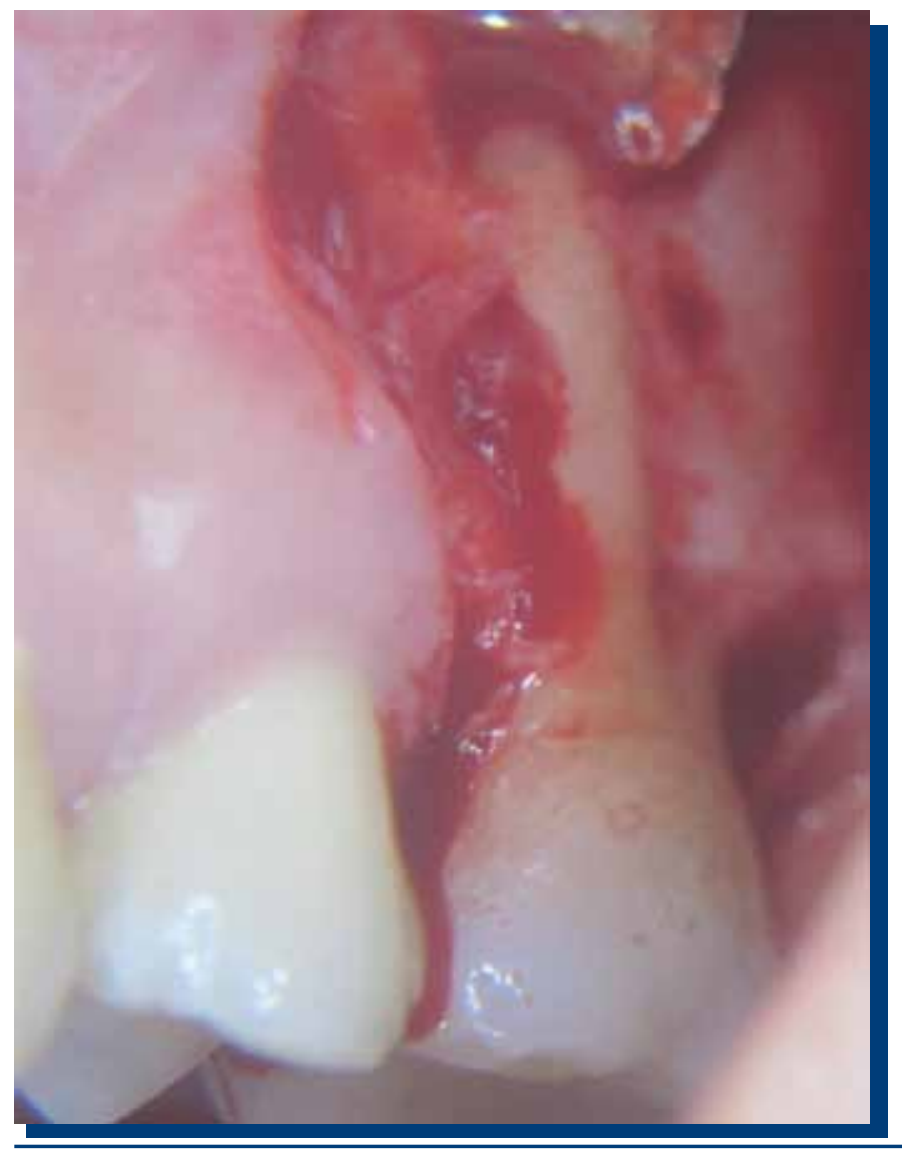

Figura 4. Imagen previa a la radectomía sin evidencia de perforación radicular. 


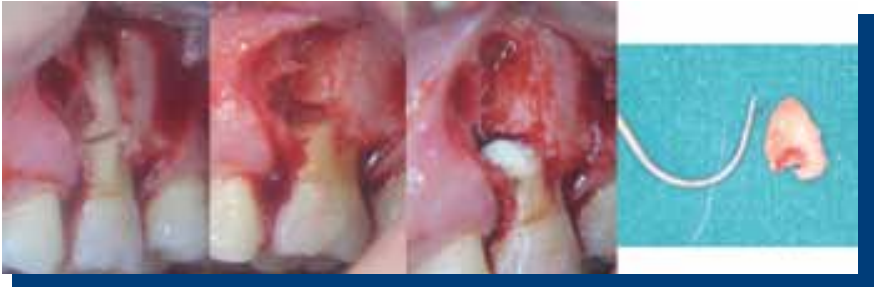

Figura 5. Radectomía raíz mesiovestibular diente 2.6.

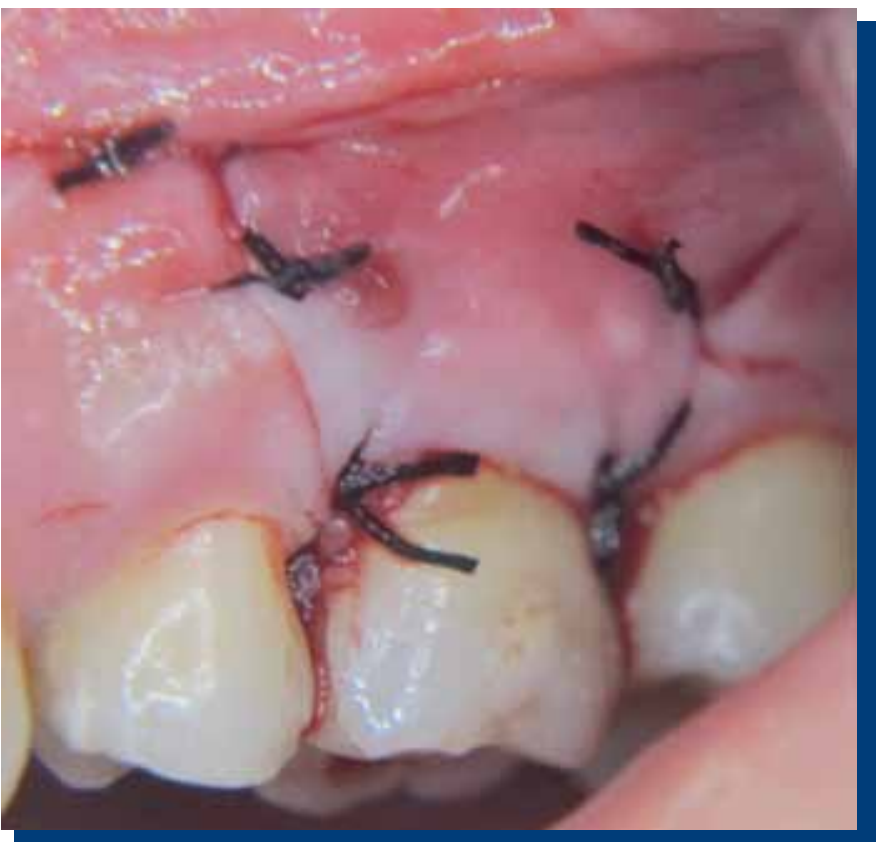

Figura 6. Imagen postoperatoria inmediata.

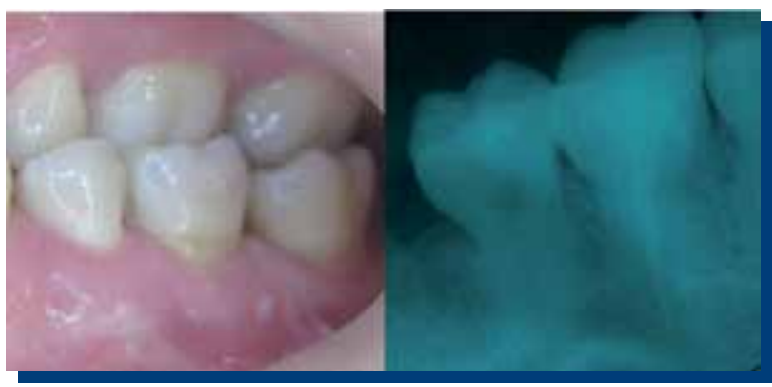

Figura 7. Control clínico y radiográfico a los 6 meses.

\section{DISCUSIÓN}

Las complicaciones asociadas a las perforaciones radiculares dependen del grado de la lesión. Cuando el contacto del microtornillo con la raíz se limita a la del espacio del ligamento periodontal, la lesión puede ser reparada sin más consecuencias ${ }^{(4)}$. Sin embargo, el daño mecánico del cemento y la resultante exposición dentinaria estimularían los procesos reabsorción radicular.

Por otro lado, si eliminamos la causa, como por ejemplo mediante la remoción del microtornillo, el proceso de reabsorción se detiene espontáneamente y la reparación de cemento con un tejido similar ocurrirá dentro de 2 a 3 semanas ${ }^{(5)}$. No obstante, una respuesta irreversible puede ocurrir cuando el área afectada es grande o la lesión es profunda. La anquilosis puede ocurrir si el área afectada es mayor a $4 \mathrm{~mm}^{2}$ ó $20 \%$ de la superficie radicular ${ }^{(2)}$. Además, la pérdida de la vitalidad del diente puede ocurrir en pacientes cuya lesión es profunda y compromete el tejido pulpar. En nuestro caso se ha producido de forma conjunta la destrucción de los tejidos periodontales y dentales en la porción lateral de la raíz mesiovestibular del primer molar superior izquierdo (área afectada mayor a $4 \mathrm{~mm}^{2}$ ) con la consecuente pérdida de vitalidad pulpar debido a la perforación de esta por un microtornillo, cumpliéndose los requerimientos en extensión y profundidad de la lesión descritos por la literatura y que pueden explicar la rizálisis sufrida por el molar.

El tiempo entre la perforación y reparación es un factor crítico para el éxito del tratamiento(6,7). En este paciente, el diagnostico de exorizalisis se realizo mínimo 2 años posterior a la realización de la perforación radicular gracias al uso de tecnología Cone Beam. Dado el tiempo de evolución de la lesión, la extensión de esta y posterior a la evaluación por un endodoncista, fue necesario un tratamiento agresivo como lo es la radectomia. Sin embargo, en casos menos severos, la literatura describe tratamientos más conservadores como lo es el manejo de la perforación radicular con MTA, el cual ha presentado excelentes resultados $^{(7)}$

Mención especial se debe dar al uso de apoyo imagenlógico adecuado, ya que gracias al uso de una tomografía digital volumétrica se pudo pesquisar la perforación radicular que no era evidente de observar con los exámenes radiológicos convencionales ni a la exploración clínica.

Algunos estudios de casos han demostrado las ventajas de la tomografía digital volumétrica sobre la radiografía convencional, no sólo en detectar la reabsorción sino también en evaluar su extensión ${ }^{(8,9-11)}$. La tomografía digital volumétrica demostró mayor sensibilidad en detectar las cavidades de reabsorción radicular externa que la radiografía panorámica convencional|(12)

Hay un consenso generalizado acerca de que con la tomografía digital volumétrica se puede conocer la localización exacta y la extensión de la reabsorción radicular así como la posible perforación y comunicación con el espacio del ligamento periodontal aportando una gran información acerca del diagnóstico, pronóstico, plan de tratamiento y seguimiento para manejar estos $\operatorname{casos}^{(8,13,14)}$.

\section{CONCLUSIÓN}

Aunque este paciente fue manejado con éxito y sin mayores complicaciones, la perforación radicular es una causa potencial de problemas legales. Se debe hacer el máximo esfuerzo para evitar daños a las raíces durante la colocación del microtornillo. Sobre todo, se debe prestar especial atención a la selección del sitio para la correcta colocación de estos. Si el sitio no cumple con los requerimientos anatómicos en términos de un posible daño radicular, se debe considerar un cambio en el lugar de colocación del tornillo, a pesar de que la mecánica de ortodoncia pueda ser bastante desfavorable.

\section{CONFLICTOS DE INTERÉS}

Los autores declaran no tener conflictos de interés. 


\section{REFERENCIAS BIBLIOGRÁFICAS}

1. Ren Y. Mini-implants for direct or indirect orthodontic anchorage. Evid Based Dent, 2009; 10(4): 113.

2. Kravitza N, Kusnotob B. Risks and complications of miniscrews. Am J Orthod Dentofacial Orthop, 2007 April; 131(4): S43-S51. Supplement.

3. Lenguas AL, Ortega R, Samara G, López MA. Tomografía computerizada de haz cónico. Aplicaciones clínicas en odontología; comparación con otras técnicas. Cient Dent, 2010; 7(2): 147-159.

4. Fabbroni G, Aabed S, Mizen K, Starr DG. Transalveolar screws and the incidence of dental damage: A prospective study. Int J Oral Maxillofac Surg, 2004; 33: 442446.

5. Asscherickx K, Vannet BV, Wehrbein H, Sabzevar MM. Root repair after injury from mini-screw. Clin Oral Implants Res, 2005; 16: 575-578.

6. Fuss Z, Trope M. Root perforations: Classification and treatment choices based on prognostic factors. Endod Dent Traumatol, 1996; 12: 255-264.

7. Menezes R, da Silva Neto UX, Carneiro E, Letra A, Bramante CM, Bernadinelli N. MTA repair of a supracrestal perforation: A case report. J Endod, 2005; 31: 212-214. 8. Cotton TP, Geisler TM, Holden DT, Schwartz SA, Schindler WG. Endodontic applications of cone-beam volumetric tomography. J Endod, 2007; 9: 1121-1132.
9. Patel S, Dadwood A. The use of cone beam computed tomography in the management of external cervical resorption lesions. Int Endod J, 2007; 40(9): 730737.

10. Maini A, Durning P, Drage N. Resorption: Within or without? The benefit of cone beam computed tomography when diagnosing a case of an internal/external resorption defect. Br Dent J, 2008; 204(3): 135-137.

11. Patel S, Kanagasingam S, Pitt Ford T. External cervical resoption: A review. J Endod, 2009; 35(5): 616-625.

12. Alqerban A, Jacobs R, Souza PC, Willems $G$. In vitro comparison of 2 cone-beam computed tomography systems and panoramic imaging for detecting simulated canine impaction-induced external root resorption in maxillary lateral incisors. Am J Orthod J Dentofacial Orthop, 2009; 136(6): 764. e1-11; discussion 764-765.

13. Patel S. New dimensions in endodontic imaging: Part. 2. Cone beam computed tomography. Int Endod J, 2009; 42(6): 463-475.

14. Tetradis S, Anstey P, Graff-Radford S. Cone beam computed tomography in the diagnosis of dental disease. J Calif Dent Assoc, 2010; 38(1): 27-32. 